\title{
Spiroplasma spp. from transmissible spongiform encephalopathy brains or ticks induce spongiform encephalopathy in ruminants
}

\author{
Correspondence \\ Frank O. Bastian \\ fbastian@agcenter.lsu.edu
}

Received 10 January 2007

Accepted 19 April 2007
Frank O. Bastian, ${ }^{1}$ Dearl E. Sanders, ${ }^{2}$ Will A. Forbes,${ }^{2}$ Sue D. Hagius, ${ }^{1}$ Joel V. Walker, ${ }^{1}$ William G. Henk, ${ }^{3}$ Fred M. Enright ${ }^{1}$ and Philip H. Elzer ${ }^{1}$

${ }^{1}$ Department of Veterinary Science, Louisiana State University Agricultural Center, 111 Dalrymple Building, Baton Rouge, LA 70803, USA

${ }^{2}$ Idlewild Research Station, Louisiana State University Agricultural Center, Baton Rouge, LA 70803, USA

${ }^{3}$ Department of Comparative Biomedical Sciences, Louisiana State University School of Veterinary Medicine, Baton Rouge, LA, USA

Spiroplasma, small motile wall-less bacteria, are linked by molecular and serological studies to the transmissible spongiform encephalopathies (TSEs), which include scrapie in sheep, chronic wasting disease (CWD) in deer and Creutzfeldt-Jakob disease in humans. In this study, two experiments were undertaken to determine the role of spiroplasma in the pathogenesis of TSE. In experiment 1, Spiroplasma mirum, a rabbit tick isolate that had previously been shown to experimentally induce spongiform encephalopathy in rodents, was inoculated intracranially (IC) into ruminants. S. mirum-inoculated deer manifested clinical signs of TSE after 1.5 to 5.5 months incubation. The deer, as well as sheep and goats, inoculated with S. mirum developed spongiform encephalopathy in a dose-dependent manner. In experiment 2 , spiroplasma closely related to S. mirum were isolated from TSE-affected brains via passage in embryonated eggs, and propagated in cell-free M1D media. Spiroplasma spp. isolates from scrapie-affected sheep brain and from CWD-affected deer brain inoculated IC into sheep and goats induced spongiform encephalopathy closely resembling natural TSE in these animals. These data show spiroplasma to be consistently associated with TSE, and able experimentally to cause TSE in ruminant animal models, therein questioning the validity of studies that have concluded the prion, a miss-folded protease-resistant protein that builds up in TSE brains during the course of the disease, to be the sole causal agent. The spiroplasma infection models reported here will be important for investigating factors involved in the pathogenesis of TSE since ruminants are the natural hosts.

\section{INTRODUCTION}

The transmissible spongiform encephalopathies (TSEs), including scrapie in sheep, chronic wasting disease (CWD) in deer and Creutzfeldt-Jakob disease (CJD) in humans, are caused by a filterable pathogen, the nature of which is controversial. Studies involving genetically altered transgenic animals have concluded that the only agent in TSE is the prion (Telling, 2000), a protease-resistant protein that builds up in TSE brains during the course of the disease.

Abbreviations: CJD, Creutzfeldt-Jakob disease; CWD, chronic wasting disease; EM, electron microscopy; IC, intracranially; SMCA, suckling mouse cataract agent; TEM, transmission electron microscopy; TSE, transmissible spongiform encephalopathy.

Supporting figures and a table are available as supplementary material with the online version of this paper.
On the other hand, there is evidence that TSE infectivity and the prion are not one and the same (Manuelidis et al., 1995; Sakaguchi et al., 1993; Xi et al., 1992). Although the prion may be important in the pathogenesis of TSE, something is missing since the prion cannot by itself induce TSE. Several recent attempts to do so have not been convincing (Legname et al., 2004; Castilla et al., 2005). Even proponents of the prion concept have suggested that other factors are involved (Kaneko et al., 1997). There is evidence suggesting that the prion may serve as a receptor protein for a bacterium (Watarai et al., 2003), which supports our hypothesis that spiroplasma, a wall-less bacterium, is a factor in the pathogenesis of TSE (Bastian, 2005). Spiroplasma-like bacteria were initially shown in CJD brains by electron microscopy (EM) (Bastian, 1979; Bastian et al., 1981; Gray et al., 1980; Reyes \& Hoenig, 1981), and spiroplasma 16S rDNA has 
been demonstrated in the majority of tested brains affected with TSE (Bastian et al., 2004). Rabbit antisera directed against scrapie-associated fibrils (Merz et al., 1983), a consistent morphological marker for TSE, also reacted with multiple spiroplasma antigens (Bastian et al., 1987a). Sera from CJD patients and sheep with scrapie had antibodies to unique spiroplasma proteins that were not present in controls (Moyer, 2004). Spiroplasma mirum has experimentally induced persistent brain infection in mice (Clark, 1974), and S. mirum infection in suckling rats is manifested by neurological deterioration and spongiform encephalopathy (Bastian et al., 1984; Tully et al., 1984). The problem is that current studies of TSE have been limited to investigations of genetically altered transgenic mice rather than studies of the behaviour of the infectious agent in its natural hosts. In this study, ruminant models, including sheep, goats and deer, were experimentally inoculated with spiroplasma; neonates were chosen since experimental TSE produces clinical disease in suckling mice with shortened incubation (McKinley et al., 1989). The spiroplasma inocula included a $S$. mirum laboratory strain, a suckling mouse cataract agent (SMCA) that had undergone repeated passages in cell-free media (Tully et al., 1977), and Spiroplasma spp. isolated from TSE-affected ruminant brains in embryonated eggs and subsequently cultured in cell-free media.

\section{METHODS}

\section{Experiment 1: SMCA strain of S. mirum inoculated intracranially (IC) into deer, sheep and goats}

An overview of experiment 1 is shown in Supplementary Fig. S1 (available with the online journal).

Inocula. A SMCA strain of S. mirum, courtesy of Dr Gail Gasparich, Towson University, Baltimore, MD, USA, was used for the inocula in experiment 1 and had undergone multiple passages in M1D broth. SMCA was grown to $\log$ phase $\left(1 \times 10^{9}\right)$ (Bastian et al., 1984), aliquoted into $1 \mathrm{ml}$ portions mixed 50/50 with sterilized $30 \%$ glycerol giving a $15 \%$ glycerol mixture and frozen at $-80^{\circ} \mathrm{C}$. After thawing, the number of organisms was presumed to be $1 \times 10^{8} \mathrm{ml}^{-1}$ as similarly prepared cultures have been enumerated by a standard tenfold titration assay (Bastian et al., 1984). The formula for M1D broth is published (Whitcomb, 1983).

Deer study. Four neonatal (5-day-old) female white-tailed deer (Odocoileus virginianus) were inoculated IC with the SMCA strain of S. mirum. Four pen-raised twin pairs of deer were used in the study: for each pair, one twin was used as a control and one was inoculated. The four inoculated neonatal deer were injected IC through the bregmatic fontanelle into the left cerebral hemisphere with $2 \mathrm{ml} \log$ phase SMCA culture in M1D media (the dose was determined to be approximately $1 \times 10^{8}$ organisms $\mathrm{ml}^{-1}$ by titration assay). The uninoculated twin control group was housed separately from the spiroplasma infection group. The deer were followed for development of neurological signs at which time the animals were anesthetized [by overdose of xylazine $\left(100 \mathrm{mg} \mathrm{ml}^{-1}\right)$ administered intravenously at a dose of $2.2 \mathrm{mg} \mathrm{kg}^{-1}$ ], exsanguinated and necropsied. All studies were done according to protocols approved by the Louisiana State University Agricultural Center Institutional Animal Care and Use Committee.
Study in sheep and goats. Other ruminants were similarly tested for susceptibility to SMCA spiroplasma infection, although they received a much lower dose of SMCA inoculum than that given to the deer. One sheep received 1/10 dilution log phase SMCA culture (approximately $1 \times 10^{7}$ organisms $\mathrm{ml}^{-1}$ ), another sheep received $1 / 100$ dilution SMCA log phase culture (approximately $1 \times 10^{6}$ organisms $\mathrm{ml}^{-1}$ ) and a control sheep received $1 \mathrm{ml} \mathrm{M1D}$ media IC; all were followed for 16.5 months. In addition, six neonatal goats were inoculated IC with $1 \mathrm{ml}$ SMCA log phase culture (approximately $1 \times 10^{8} \mathrm{ml}^{-1}$ ). Of these, five animals were euthanized at 2 months; 1 animal was euthanized at 4 months. Two control goats inoculated IC with M1D media alone were necropsied, one at 2 months and one at 4 months.

Pathological studies. At necropsy, the brains were split sagitally upon removal, the right half was frozen at $-20{ }^{\circ} \mathrm{C}$ and the other half fixed in $20 \%$ buffered formalin for 2 weeks. The fixed brain was sectioned at the juncture of brain stem and cerebrum, and the cerebellum was separated from the brain stem. Each portion was individually weighed and compared one to another. The cerebella from the spiroplasma-inoculated ruminants were consistently smaller by weight than the normal controls. Standard sections were taken from each portion, and included cerebral cortex, hippocampus, basal ganglia, upper brain stem, lower brain stem including obex, cerebellum and spinal cord; all sections were stained with haematoxylin and eosin. The hippocampi were carefully examined for evidence of hypoxia or autolysis. These procedures applied to all ruminant animals studied.

Immunohistochemistry. In the deer study where the laboratory strain SMCA spiroplasma had been inoculated, immunohistochemistry using standard procedures (Bastian et al., 1984) was carried out in regions of the brain stem of deer 2 where lesions were identified by light microscopy. The primary antibody against SMCA (courtesy of Dr Gail Gasparich, Towson University, Baltimore, MD, USA) had been generated in rabbits by direct inoculation of the organism, since spiroplasma does not induce disease in adult rabbits (Kirchhoff et al., 1981).

EM. The site in the brain stem of deer 2 where immunocytochemistry had revealed the presence of spiroplasma antigens was further studied by transmission EM (TEM). A small $1 \mathrm{~mm}$ cube sampling was obtained from brain tissues just below the floor of the fourth ventricle, where extensive vacuolization had been noted on light microscopy, and organisms had been found by immunohistochemistry. These samples were post-fixed in $1.25 \%$ glutaraldehyde, $2 \%$ formaldehyde, $0.1 \mathrm{M}$ cacodylate buffer ( $\mathrm{pH}$ 7.4) for $1 \mathrm{~h}$ and processed for EM using standard methodology. Vacuolated neurons were identified on certain of the thick Epon sections, and adjacent thin sections were cut from those Epon blocks and examined using a JEOL electron microscope (JEM 1011).

Experiment 2: Spiroplasma spp. were isolated via passage in embryonated eggs from TSE-infected brains then inoculated IC into ruminants

An overview of experiment 2 is shown in Supplementary Fig. S1 (available with the online journal).

TSE-affected and normal brain samples. Frozen scrapie-affected sheep brain samples were obtained courtesy of Katherine O'Rourke, USDA, Pullman, WA, USA, and CWD-affected deer brain frozen samples were obtained courtesy of Terry Spraker, Colorado State Veterinary Diagnostic Laboratory, Colorado State University, Fort Collins, CO 80523, USA. Frozen normal sheep brains were obtained from the Department of Veterinary Science, Louisiana State University Agricultural Center. Normal deer brains were obtained 
from a hunting lodge in Alabama. CJD-affected brains and normal age-matched human brains were obtained from the necropsy service and the CJD-Diagnostic Center at Tulane Health Science Center, New Orleans, LA, USA.

Spiroplasma cultured via passage through embryonated eggs. Approximately 250 mg scrapie-, CWD-, or CJD-affected brain tissues, or normal sheep or deer brain controls, were homogenized in $3 \mathrm{ml}$ M1D media (Whitcomb, 1983), centrifuged at $10000 \mathrm{~g}$ for $1 \mathrm{~min}$ and the supernatant filtered $(220 \mathrm{~nm})$ then inoculated into the yolk sac of 8-day-old pathogen-free embryonated eggs (Charles River Laboratories). Ten eggs were inoculated each with $100 \mu$ l aliquots of the supernatant derived from the TSE-affected brain. Following 9 days incubation at $37^{\circ} \mathrm{C}$ in a humidified incubator, the egg shell over the air sac was removed, and the amniotic and allantoic fluids were aspirated and added directly to $5 \mathrm{ml}$ fresh M1D broth, then the tubes were stoppered and placed in a humidified incubator at $37^{\circ} \mathrm{C}$. Vancomycin $\left(30 \mu \mathrm{g} \mathrm{ml}^{-1}\right)$ was added to each tube to prevent bacterial contamination. Cultures were blind passaged every two weeks by mixing $50 / 50$ with fresh M1D media and examined biweekly using phase microscopy at $\times 1000$ (oil emersion) for the presence of motile helices. At 2 months post-inoculation, when bacteria appeared plentiful by phase microscopy, $2 \mathrm{ml}$ culture was centrifuged at $14000 \mathrm{~g}$ for $20 \mathrm{~min}$, and the pellet examined morphologically and molecularly for the presence of spiroplasma. Samples of the initial passages from each TSE case were frozen in $1 \mathrm{ml}$ aliquots 50/50 with $30 \%$ sterile glycerol for future inoculation into ruminants. Control eggs were inoculated either with M1D media alone or with normal sheep, deer or human brain homogenates processed as above for the TSE-affected samples. The controls were similarly examined by phase microscopy and molecular studies for the presence of spiroplasma.

EM studies of egg-passaged cultures. Spiroplasma were searched for in the cell-free broth obtained from the TSE-affected brains via passage through embryonated eggs by both TEM and negative stain EM. At 2 months of incubation, when phase microscopy revealed sufficient numbers of motile helices, a centrifuged pellet was fixed in $3 \%$ buffered glutaraldehyde and processed as above for TEM. Also a drop of the concentrated culture was air-dried on a Formvar-coated grid and prepared for negative stain EM. The EM studies used standard methodology.

Molecular studies. Molecular identification of spiroplasma was possible since our discovery of spiroplasma-specific oligonucleotide primers sets for PCR (Bastian et al., 2004). DNA was extracted using standard phenol/chloroform methodology from a centrifuged pellet of the cell-free culture containing the isolate described above. PCR was carried out using oligonucleotide primers that specifically identified a 270 bp portion of S. mirum-related $16 \mathrm{~S}$ rDNA (Bastian et al., 2004) (Supplementary Fig. S2, Supplementary Fig. S3 available with the online journal). The DNA from the amplified PCR product was extracted and cloned using standard methodology then submitted for DNA sequencing. BLAST analysis of the sequence on the GenBank database established the relationship of the spiroplasma isolated to $S$. mirum (Supplementary Fig. S3 available with the online journal).

Inoculation into ruminants. The cell-free broth isolates of Spiroplasma spp. obtained from scrapie- or CWD-affected brains via passage in embryonated eggs were then inoculated IC into sheep and goats. The titre of the inocula could not be determined since the Spiroplasma spp. derived from the egg passage showed fastidious growth and had not been adapted to growth on agar. Three neonatal sheep were inoculated IC with $1 \mathrm{ml}$ Spiroplasma spp. isolated from scrapie-affected sheep brain and another neonatal sheep was inoculated IC with $1 \mathrm{ml}$ spiroplasma isolated from CWD-affected deer brain. The sheep were euthanized and necropsied at 11 months. Four neonatal goats were inoculated IC with $1 \mathrm{ml}$ Spiroplasma spp. isolated from scrapie-affected sheep brain, and were euthanized and necropsied at 12 months. In addition, five neonatal goats were inoculated IC with $1 \mathrm{ml}$ Spiroplasma spp. isolated from CWD-affected deer brain and were euthanized and necropsied at 11 months. One neonatal goat was inoculated IC with $1 \mathrm{ml} \mathrm{M} 1 \mathrm{D}$ media and was euthanized at 11 months.

Prion detection. The CJD spiroplasma isolate preparations were tested for the presence of abnormal prion proteins using methodology recently developed in our laboratory. Protein was extracted from a centrifuged pellet of the culture using guanidine (Bastian et al., 2005) and tested for presence of prions by Western blotting using the 3F4 anti-prion mAb.

\section{RESULTS AND DISCUSSION}

\section{Experimental infection with SMCA}

In experiment 1 , three of four deer inoculated with spiroplasma developed clinical signs of neurological deterioration, while the controls remained clinically normal. In the SMCA infection group, deer 1 developed dysphagia and stopped eating at 1.5 months. The veterinarian examining the deer considered the clinical signs to be neurological in origin, and necropsy findings supported his assessment (Supplementary Fig. S4 available with the online journal). Deer 2 at 3.5 months was unable to stand or walk, startled easily and stopped eating. She developed a constant grinding of the teeth (bruxism), which was indicative of degenerative brain disease and has been seen in natural scrapie in ruminants (Hadlow, 1961; Haig \& Clarke, 1965). Her ears were laid back, her tail remained tucked tightly over the perineal region and her rumen at necropsy was dilated and water-filled, indicating polydipsia. Her rib cage was prominent suggesting significant wasting. One week prior to the onset of clinical disease she had appeared normal. The rapid progression of these types of clinical signs was typical of CWD (Spraker et al., 1997). Deer 3 developed signs of ataxia at 5.5 months and was necropsied. The fourth SMCA inoculated deer (deer 4) was also necropsied at 5.5 months with no evidence of clinical disease. The four un-inoculated control deer showed no clinical signs of disease during the course of the experiment; two control deer were necropsied at 4.5 months and two were necropsied at 5.5 months at the termination of the experiment.

All deer brains were uniformly examined at necropsy to determine the location and nature of any lesions. For the most part, the spiroplasma-inoculated deer brains showed no gross anomalies, except in deer 3 where the brain showed marked hydrocephalus. The cerebella in each of the spiroplasma-inoculated deer were smaller by weight compared to cerebella of the normal control deer. The haematoxylin and eosin stained sections revealed spongiform encephalopathy in the cerebral cortex, cerebellar cortex and the brain stem of spiroplasma-infected deer, while brains from corresponding control deer were histologically normal. The SMCA-inoculated deer 1 showed early changes of spongiform encephalopathy with 
vacuolization of the neuropil in the cerebral cortex and cerebellar cortex (Supplementary Fig. S4 available with the online journal). The SMCA-inoculated deer 2 examined 2 months later than deer 1 showed prominent vacuolization of the neuropil in the brain stem (Fig. 1a) and more severe vacuolization of the cerebellar cortex (Fig. 1b), indicating progression of the disease process. Spiroplasma were identified in the brain stem of deer 2 beneath the floor of the fourth ventricle by immunocytochemistry (Fig. 1c) and by TEM (Fig. 1d, e). All deer inoculated with S. mirum developed spongiform encephalopathy, while the control deer remained normal. In a follow up control experiment, 5 deer were inoculated IC at 2 months of age with $1 \mathrm{ml}$ M1D media and showed no clinical signs of disease at 8 months.

Other ruminants tested showed susceptibility to SMCA spiroplasma infection. While sheep inoculated IC with the SMCA laboratory strain lacked clinical signs of disease through 16.5 months of observation, upon necropsy, histological examination revealed spongiform encephalopathy dependent upon the dose of inoculum. One sheep that received $1 / 10$ dilution of the log phase SMCA culture (approximately $1 \times 10^{7}$ organisms $\mathrm{ml}^{-1}$ ) showed severe spongiform degeneration of the hippocampus, cerebellum and brain stem (Fig. 2a). Another sheep receiving 1/100 dilution of the SMCA log phase culture (approximately $1 \times 10^{6}$ organisms $\mathrm{ml}^{-1}$ ) showed a lesser degree of spongiform alteration of the cerebellum (Fig. 2b). The control sheep received $1 \mathrm{ml}$ M1D media IC and showed no pathology at 16.5 months (Fig. 2c). All SMCA-inoculated goats showed minimal vacuolization of the neuropil in comparison to the controls (data not shown); the lesions were consistent with the early sampling in the experiment. The hippocampi were well preserved in all instances, lacking evidence of hypoxia or autolysis indicating rapid fixation of the brains following necropsy. Therefore, sheep and goats were susceptible to experimental SMCA spiroplasma infection and developed a progressive spongiform encephalopathy.

\section{Isolation of Spiroplasma spp. from TSE-affected brains}

In the second series of experiments, Spiroplasma spp. were isolated from brains of sheep affected with scrapie, CWDaffected deer brains and CJD-affected human brains via passage in embryonated eggs followed by cultivation in cell-free M1D media. The rationale for this approach was

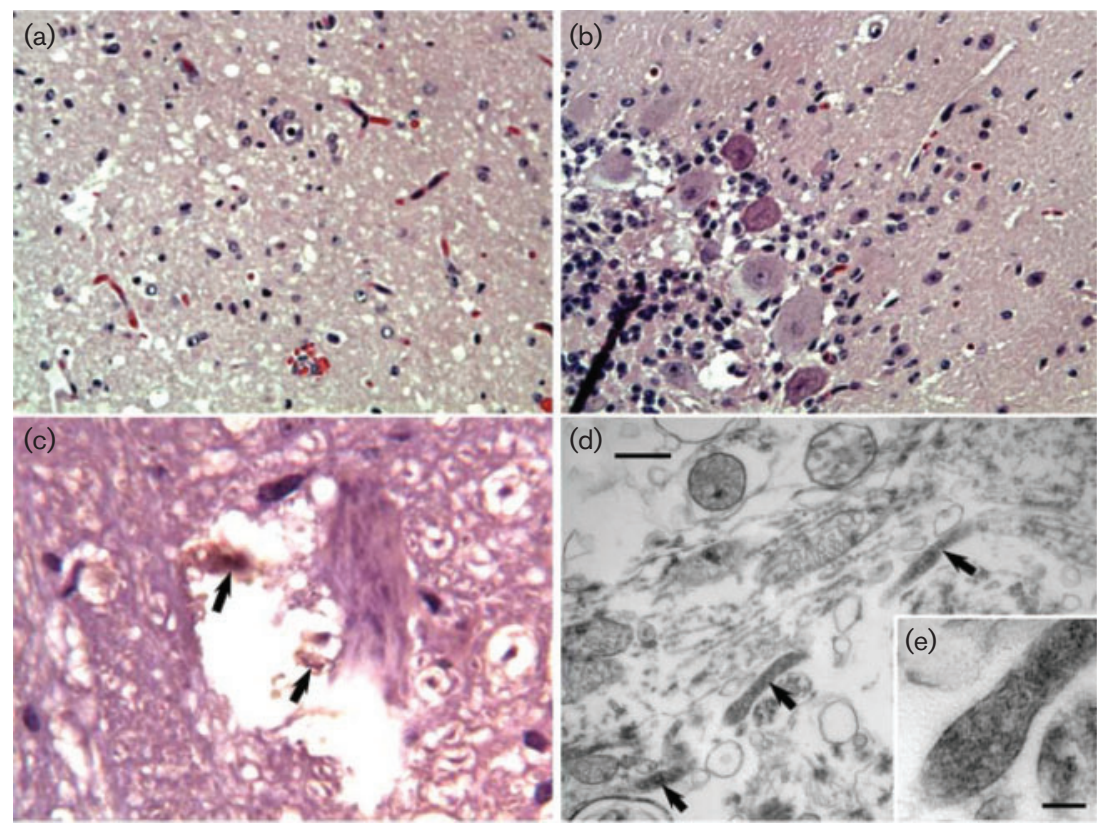

Fig. 1. Experimental inoculation of a SMCA strain of S. mirum induced spongiform encephalopathy in four deer tested. (a) The brain stem and (b) cerebellar cortex from deer 2 showed spongiform encephalopathy. Note the peripheral cytoplasmic vacuolization of the Purkinje neurons of the cerebellar cortex and microglial infiltration of the overlying molecular layer. There is depletion of granular cells and prominence of Bergman astrocytes, all representing histological findings typical of TSE. Magnification $\times 400$. (c) Spiroplasma organisms (indicated by arrows) were demonstrated within a neuronal vacuole in the brain stem of deer 2 by immunohistochemistry using SMCA-specific rabbit sera as primary antibody. Note the absence of inflammation. Magnification $\times 800$. (d) A spiroplasma at least $4 \mu \mathrm{m}$ long was demonstrated within a cell process by TEM. Arrows indicate spiroplasma. Bar, $500 \mathrm{~nm}$. (e) The inset shows the organism at high magnification measuring less than $100 \mathrm{~nm}$ in diameter. Bar, $100 \mathrm{~nm}$. 


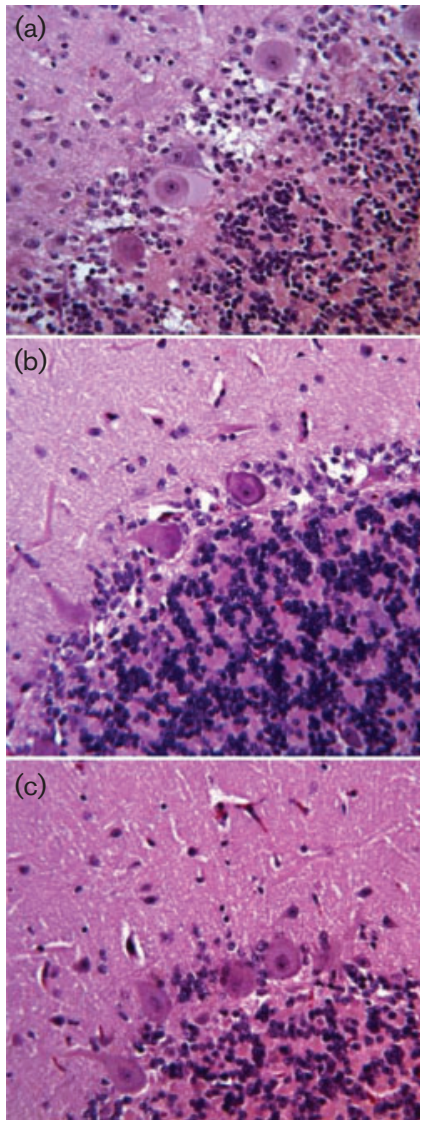

Fig. 2. Sheep inoculated IC with SMCA with varying titres of inoculum. (a) A sheep brain inoculated with a 1/10 dilution $\left(1 \times 10^{7}\right)$ of log phase SMCA culture showed at 16.5 months spongiform degeneration of the cerebellar cortex with Bergmann astrocyte proliferation, depletion of granular cell neurons and microglia infiltrate in the molecular layer. (b) Sheep inoculated with $1 / 100$ dilution $\left(1 \times 10^{6}\right)$ of log phase SMCA culture showed at 16.5 months less advanced spongiform change with vacuolization of the cerebellar cortex in the Purkinje neurons. (c) The cerebellar cortex of control sheep inoculated IC with media examined at 16.5 months showed no pathology. The evolution of spongiform lesions seen in the sheep with a ten-fold increase in inocula titre represents a dose-dependent pathological response, which corroborates our evidence of dose response to experimental $S$. mirum infection in the rat model (Bastian et al., 1984). These data correlate with sub-clinical experimental scrapie infections wherein early spongiform changes are seen midway through the incubation period (Hadlow, 1961; Race et al., 2002). Magnification $\times 400$.

that the initial isolation of SMCA from mice inoculated with homogenized rabbit ticks used this methodology (Clark, 1974). The TSE-affected brains from which the spiroplasma isolations were obtained had previously been documented to contain spiroplasma $16 \mathrm{~S}$ rDNA by molecular studies (Bastian et al., 2004). After 9 days incubation at $37^{\circ} \mathrm{C}$, the TSE-inoculated embryonated eggs revealed under-developed or dead embryos, while the eggs inoculated with media alone or normal brain tissue had normal developing embryos. After transfer of the allantoic fluid from the eggs into the cell-free broth, the cultures derived from the TSE-inoculated eggs showed acidification after 16 days incubation at $37^{\circ} \mathrm{C}$, at which time phase microscopy $(\times 1000)$ revealed motile helices. Further growth of the Spiroplasma spp. from the TSE brains required blind passages every 2 weeks for 2 months until sufficient numbers of spiroplasma were identified by phase microscopy to allow EM and molecular studies. Spiroplasma spp. were identified in the cultures both by negative stain EM (Fig. 3a) and TEM (Fig. 3b). The helical wall-less bacteria measured only $60 \mathrm{~nm}$ in diameter (Fig. 3b). The spiroplasma TSE isolates were further identified by PCR and DNA sequence analysis as being closely related to $S$. mirum (Supplementary Fig. S2, Supplementary Fig. S3 available with the online journal). All seven TSE-affected brains passaged via embryonated eggs resulted in growth of spiroplasma [scrapie-affected sheep brains (2/2), CWD-affected deer brain (1/1), and CJD-affected human brains (4/4)] in cell-free broth, while

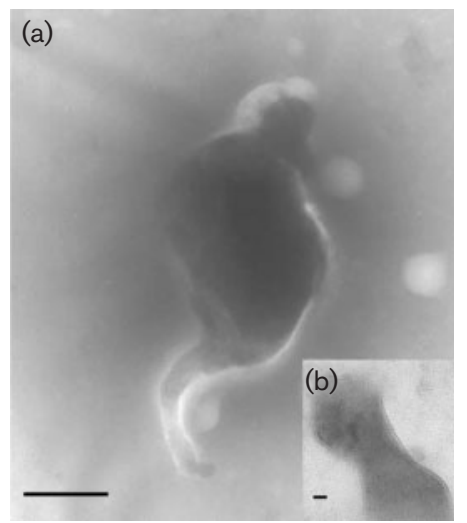

Fig. 3. Spiroplasma spp. isolated from TSE-affected brains via passage in embryonated eggs. (a) Two entwined helical spiroplasma derived from CJD brain tissues via passage in embryonated eggs are demonstrated by negative stain EM in a cell-free culture. These CJD Spiroplasma spp. isolates were morphologically identical to spiroplasma isolated from scrapie-affected sheep brains as shown by negative stain EM in cell-free broth (Supplementary Fig. S5 available with the online journal). Bar, 500 $\mathrm{nm}$. (b) Spiroplasma spp. were also identified by TEM of the pellet of cell-free broth culture derived from egg-passaged scrapieaffected sheep brain. Bar, $20 \mathrm{~nm}$. The organism, sectioned obliquely, was seen as a wall-less bacterium that measured 60$100 \mathrm{~nm}$ in diameter. This ultrastructural study confirmed that Spiroplasma spp. had been isolated from the TSE-affected brains via passage in embryonated eggs; further identification of the spiroplasma isolates as being closely related to $S$. mirum was determined by PCR and DNA sequence analysis (Supplementary Figs S2 and S3 available with the online journal) (Bastian et al., 2004). The ability to isolate Spiroplasma spp. from essentially all TSE-affected brains tested (Supplementary Table S1 available with the online journal) is significant since it will enhance further investigations of TSE causality and pathogenesis. 
passage of control normal brains [including human brains $(0 / 3)$, sheep brains $(0 / 3)$ and deer brains $(0 / 3)]$ or media alone showed no evidence of spiroplasma growth (Supplementary Table S1 available with the online journal). These studies represent compelling evidence for the presence of spiroplasma in TSE.

\section{Experimental infection with Spiroplasma spp. isolated from TSE-affected brains}

While none of the ruminants inoculated with the TSE spiroplasma isolates developed clinical signs of disease, all showed pathology at necropsy. In natural scrapie, pathology in the brain typically is seen midway through the incubation period (Hadlow, 1961; Race et al., 2002). At 11 months, one goat and one sheep, each inoculated IC with spiroplasma derived from scrapie-affected sheep brain, showed severe cerebellar and hippocampal spongiform degeneration with neuronal vacuolization in the brain stem (Fig. 4a, b, c, d). There was vacuolization of the cerebellar white matter in the spiroplasma-inoculated goat (Supplementary Fig. S5 available with the online journal); cerebellar white matter involvement has been reported in both natural scrapie in goats and experimental scrapie in mice (Haig \& Clarke, 1965). The other sheep and goats inoculated with the scrapie spiroplasma isolates showed a lesser degree of spongiform change. Such variation of clinical signs and pathology is typical among ruminants experimentally inoculated with natural scrapie (Hadlow, 1961; Haig \& Clarke, 1965). Another goat inoculated with spiroplasma derived from a CWD-affected deer brain showed spongiform change involving the cerebral cortex, cerebellum and brain stem (Fig. 4e, f). A control goat and sheep inoculated IC with M1D media alone revealed no pathology at 11 months (data not shown), indicating that lesions in the spiroplasma-inoculated animals were due to the presence of the organism. It is noteworthy that the intraneuronal vacuoles in the cerebellar cortex and brain stem seen in the Spiroplasma spp. inoculated ruminants were identical to lesions typical of naturally occurring TSE (Hadlow, 1961; Spraker et al., 1997).

\section{Experimental spiroplasma infection in ruminants: a model for TSE}

The susceptibility of ruminants, natural hosts of TSE, to experimental spiroplasma infection, raises the issue of the role of spiroplasma in the pathogenesis of TSE. The similarities of the clinical spiroplasma infection in deer to natural TSE were striking. Deer 2 after 3.5 months incubation suddenly developed clinical signs consistent with CWD. Such rapid clinical deterioration has been seen in experimental scrapie infection in goats (Hadlow, 1961) and in sporadic CJD in humans (Bastian, 1991). The incubation period in the spiroplasma-infected ruminants appeared directly related to dose. This dose response was evident in studies of sheep inoculated with different dose dilutions of SMCA. The pathological studies of the

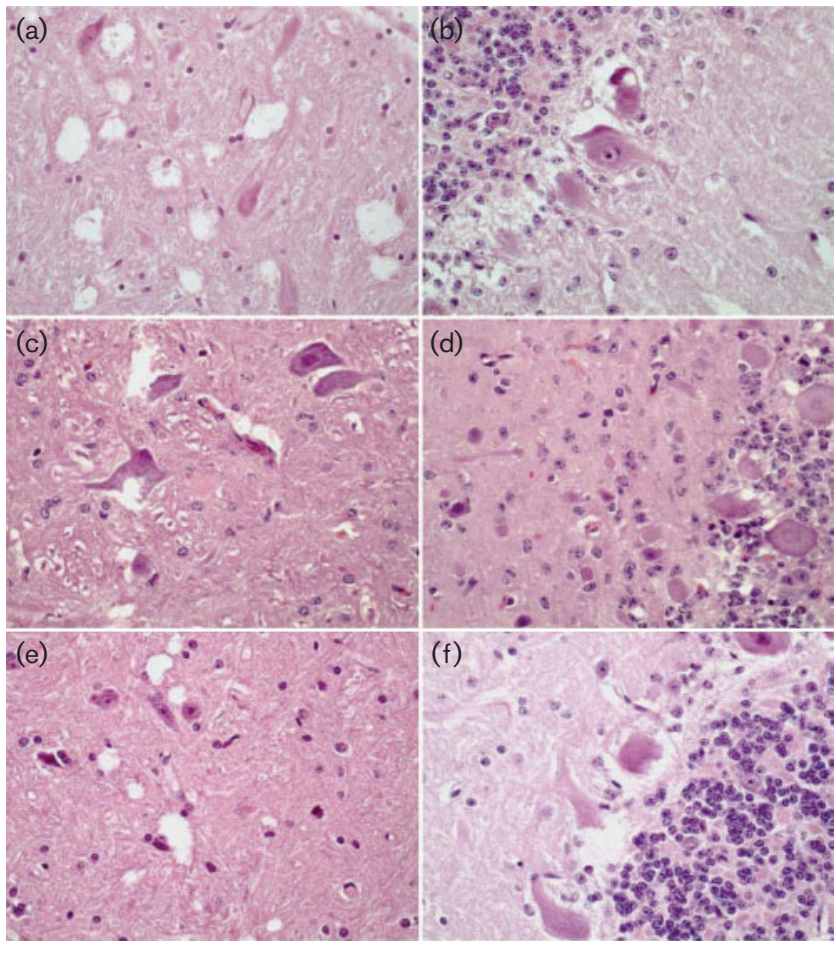

Fig. 4. Spiroplasma spp. isolated from scrapie-affected sheep brain or CWD-affected deer brain via passage in embryonated eggs induced spongiform encephalopathy in sheep and goats. (a) The brain stem and (b) cerebellar cortex from a goat inoculated IC with Spiroplasma sp. isolated from scrapie-affected sheep brain showed spongiform encephalopathy at 11 months. Note the intraneuronal vacuolization seen involving neurons in the brain stem and Purkinje neurons in the cerebellar cortex that are typical of lesions seen in natural scrapie infection in ruminants (Hadlow, 1961). (c) The brain stem and (d) cerebellar cortex from a sheep inoculated with a scrapie spiroplasma isolate showed similar histological changes as (a) and (b). (e) The brain stem and ( $f$ ) cerebellar cortex at 11 months from a goat inoculated IC with Spiroplasma sp. isolated from a deer brain affected with CWD showed intraneuronal vacuolization characteristic of natural CWD (Spraker et al., 1997). The remarkable close resemblance of lesions induced by Spiroplasma spp. isolated from TSE-affected ruminant brains to natural TSE in these same animals strongly suggest ruminants as the preferred experimental models for study of TSE pathogenesis. Magnification $\times 400$.

spiroplasma-infected ruminants showed remarkable similarities to natural TSE infections in these same hosts, both in distribution of lesions and manifestation of the pathological lesion. Other causes of spongiform encephalopathy were considered but ruled out since the controls, which were receiving the same food and water, and were maintained in a manner similar to the spiroplasmainoculated animals, remained normal and demonstrated no significant gross or histological evidence of exposure to toxicants or other infectious agents. When inoculated with the Spiroplasma spp. isolates derived from TSE-affected 
brains, the sheep and goats exhibited intracytoplasmic neuronal vacuoles that are pathognomonic of scrapie or CWD (Hadlow, 1961; Spraker et al., 1997). Spiroplasma were demonstrated at the site of these lesions in the deer brain stem by immunohistochemistry and EM consistent with their direct involvement in the pathological process. Of great significance is that spiroplasma isolated from TSEaffected brains, and inoculated into sheep and goats, induced TSE. Other routes of induction of spiroplasma infection should be tried in these ruminant models, although prior peripheral inoculation studies in the rat model have shown S. mirum to be neurotropic (Bastian et al., 1987b).

It will be important to determine if prion is deposited in brains of ruminants wherein spiroplasma has induced TSE. Prion was not found in Spiroplasma spp. cell-free broth preparations derived from CJD brain by Western blotting, suggesting that prions were removed through egg passage and subsequent repeated dilutions of the cell-free broth. In studies carried out in the 1980s, we showed that polyclonal rabbit antisera prepared against ME7 scrapie infectionspecific proteins (courtesy Dr Rubenstein, Institute for Basic Research in Developmental Disabilities, 1050 Forest Hill Road, Staten Island, NY 10314, USA) cross-reacted with detergent/protease-treated spiroplasma proteins derived from broth culture by Western blot (Bastian et al., 1987a). However, we were not able to definitively identify prions in the preparations since prion $\mathrm{mAbs}$ were not available to us at that time. One potential problem in resolving this issue is that animals inoculated with materials derived from a different animal species require adaptation of the transmissible agent to the tissues by serial passage before prions are formed (Manuelidis et al., 1997; Lasmèzas et al., 1997; Race et al., 2002). The resolution of this issue may not be determined until future experiments are carried out using serial passages in the ruminant TSE model.

In conclusion, spiroplasma, whether a multi-passaged laboratory strain originally isolated from ticks or a novel strain isolated from TSE-affected sheep or goat brains via passage in embryonated eggs, induced spongiform encephalopathy in ruminants when inoculated IC. These data provide the impetus for future epidemiological studies to determine the frequency of the association of spiroplasma with TSE. This effort will require identification of spiroplasma by serological reactivity in natural TSE cases with spiroplasma antigens (Moyer, 2004), by molecular probes of TSE-affected tissues for spiroplasma ribosomal DNA (Bastian et al., 2004), or by direct isolation of spiroplasma from TSE-affected brains as reported in this study. Molecular studies may be capable of elucidating the variability of spiroplasma strains involved in TSE as inferred from a prior study (Bastian et al., 2004). Nonetheless, this study emphasizes the importance of future TSE experiments utilizing the ruminant model that will provide needed information regarding the role and interaction of a variety of factors involved in TSE pathogenesis, including spiroplasma and the prion.

\section{ACKNOWLEDGEMENTS}

We thank W. Wimley for his encouragement and critical review, G. Gasparich for providing stocks of spiroplasma DNA and SMCA culture for inoculation, K. O'Rourke for providing samples of scrapieaffected sheep brains and T. Spraker for providing samples of CWDaffected deer brains. This work was supported in part by NIH grant R01-NS044003, donations from CJD-affected families and Louisiana State University Agricultural Center Research funds.

\section{REFERENCES}

Bastian, F. O. (1979). Spiroplasma-like inclusions in CreutzfeldtJakob disease. Arch Pathol Lab Med 103, 665-669.

Bastian, F. O. (1991). Creutzfeldt-Jakob and Other Transmissible Spongiform Encephalopathies. New York: Mosby-Year Book.

Bastian, F. O. (2005). Spiroplasma as a candidate causal agent of transmissible spongiform encephalopathies. J Neuropathol Exp Neurol 64, 833-838.

Bastian, F. O., Hart, M. N. \& Cancila, P. A. (1981). Additional evidence of spiroplasma in Creutzfeldt-Jakob disease. Lancet 1, 660.

Bastian, F. O., Purnell, D. M. \& Tully, J. G. (1984). Neuropathology of spiroplasma infection in the rat brain. Am J Pathol 114, 496-514.

Bastian, F. O., Jennings, R. A. \& Gardner, W. A. (1987a). Antiserum to scrapie-associated fibril protein cross-reacts with Spiroplasma mirum fibril protein. J Clin Microbiol 25, 2430-2431.

Bastian, F. O., Jennings, R. A. \& Hoff, C. J. (1987b). Neurotropic response of Spiroplasma mirum following peripheral inoculation in the rat. Ann Inst Pasteur Microbiol 138, 651-655.

Bastian, F. O., Dash, S. \& Garry, R. (2004). Linking chronic wasting disease to scrapie by comparison of Spiroplasma mirum ribosomal DNA sequences. Exp Mol Pathol 77, 49-56.

Bastian, F. O., McDermont, M. E., Perry, A. S., Carver, L. A., Dash, S. \& Garry, R. F. (2005). Safe method for isolation of prion protein and diagnosis of Creutzfeldt-Jakob disease. J Virol Methods 130, 133-139.

Castilla, J., Saá, P., Hetz, C. \& Soto, C. (2005). In vitro generation of infectious scrapie prions. Cell 121, 195-206.

Clark, H. F. (1974). The suckling mouse cataract agent (SMCA). Prog Med Virol 18, 307-322.

Gray, A., Francis, R. J. \& Scholtz, C. L. (1980). Spiroplasma and Creutzfeldt-Jakob disease. Lancet 2, 152.

Hadlow, W. J. (1961). The pathology of experimental scrapie in the dairy goat. Res Vet Sci 2, 289-314.

Haig, D. A. \& Clarke, M. C. (1965). Observations on the agent of scrapie. In NINDB Monograph no. 2, Slow, Latent and Temperate Virus Infections, pp. 215-219. Edited by D. C. Gajdusek, C. J. Gibbs Jr \& M. Alpers. Bethesda, MA: Public Health Service.

Kaneko, K., Zulianello, L., Scott, M., Cooper, C. M., Wallace, A. C., James, T. L., Cohen, F. E. \& Prusiner, S. B. (1997). Evidence for protein $\mathrm{X}$ binding to a discontinuous epitope on the cellular prion protein during scrapie propagation. Proc Natl Acad Sci U S A 94, 10069-10074.

Kirchhoff, H., Heitmann, J. \& Trautwein, G. (1981). Pathogenicity of Spiroplasma sp. strain SMCA in rabbits: clinical, microbiological and histological aspects. Infect Immun 33, 292-296.

Lasmèzas, C. I., Deslys, J. P., Robain, O., Jaegly, A., Beringue, V., Peyrin, J. M., Fournier, J. G., Hauw, J. J., Rossier, J. \& Dormond, D. (1997). Transmission of the BSE agent to mice in the absence of detectable abnormal prion protein. Science 275, 402-405. 
Legname, G., Baskakov, I. V., Nguyen, H.-O. B., Riesner, D., Cohen, F. E., DeArmond, S. J. \& Prusiner, S. B. (2004). Synthetic mammalian prions. Science 305, 673-676.

Manuelidis, L., Sklaviadis, T., Akowitz, A. \& Fritch, W. (1995). Viral particles are required for infection in neurodegenerative CreutzfeldtJakob disease. Proc Natl Acad Sci U S A 92, 5124-5128.

Manuelidis, L., Fritch, W. \& Xi, Y. G. (1997). Evolution of a strain of CJD that induces BSE-like plaques. Science 277, 94-98.

McKinley, M. P., DeArmond, S. J., Torchia, M., Mobley, W. C. \& Prusiner, S. B. (1989). Acceleration of scrapie in neonatal Syrian hamsters. Neurology 39, 1319-1324.

Merz, P. A., Somerville, R. A., Wisniewski, H. M., Manuelidis, L. \& Manuelidis, E. E. (1983). Scrapie-associated fibrils in CreutzfeldtJakob disease. Nature 306, 474-476.

Moyer, P. (2004). Spiroplasma hsp60 may be the pathogen responsible for spreading CJD. Neurology Today 4, 8-11.

Race, R., Meade-White, K., Raines, A., Raymond, G. J., Caughey, B. \& Chesebro, B. (2002). Subclinical scrapie infection in a resistant species: persistence, replication and adaptation of infectivity during four passages. J Infect Dis 186 (Suppl. 2), S166-S170.

Reyes, J. M. \& Hoenig, E. M. (1981). Intracellular spiral inclusions in cerebral cell processes in Creutzfeldt-Jakob disease. J Neuropathol Exp Neurol 40, 1-8.

Sakaguchi, S., Katamine, S. \& Yamanouchi, K. (1993). Kinetics of infectivity are dissociated from $\operatorname{PrP}$ accumulation in salivary glands of
Creutzfeldt-Jakob disease agent-inoculated mice. J Gen Virol 74, 2117-2123.

Spraker, T. R., Miller, M. W., Williams, E. S., Getzy, D. M., Adrian, W. J., Schoonveld, G. G., Spowart, R. A., O'Rourke, K. I., Miller, J. M. \& Merz, P. A. (1997). Spongiform encephalopathy in free-ranging mule deer (Odocoileus hemionus), white-tailed deer (Odocoileus virginianus) and Rocky Mountain Elk (Cervus elaphus nelsoni) in north central Colorado. J Wildl Dis 33, 1-6.

Telling, G. C. (2000). Prion protein genes and prion diseases: studies in transgenic mice. Neuropathol Appl Neurobiol 26, 209-212.

Tully, J. G., Whitcomb, R. F., Clark, H. F. \& Williamson, D. L. (1977). Pathogenic mycoplasma: cultivation and vertebrate pathogenicity of a new spiroplasma. Science 195, 892-894.

Tully, J. G., Bastian, F. O. \& Rose, D. L. (1984). Localization and persistence of spiroplasmas in an experimental brain infection in suckling rats. Ann Microbiol (Paris) 135A, 111-117.

Watarai, M., Kim, S., Erdenebaatar, J., Makino, S.-I., Horiuchi, M., Shirahata, T., Sakaguchi, S. \& Katamine, S. (2003). Cellular prion protein promotes Brucella infection into macrophages. J Exp Med 198, 5-17.

Whitcomb, R. F. (1983). Culture media for spiroplasmas. In Methods in Mycoplasmology, pp. 147-158. Edited by S. Razin \& J. G. Tully. New York: Academic Press.

Xi, Y. G., Ingrosso, L., Ladogana, A., Masullo, C. \& Pocchiarri, M. (1992). Amphotericin B treatment dissociates in vivo replication of the scrapie agent from PrP accumulation. Nature 356, 598-599. 